\title{
PROJECTIVELY EQUIVALENT METRICS SUBJECT TO CONSTRAINTS
}

BY

WILLIAM TABER

\begin{abstract}
This work examines the relationship between pairs of projectively equivalent Riemannian or Lorentz metrics $g$ and $g^{*}$ on a manifold $M$ that induce the same Riemannian metric on a hypersurface $H$. In general such metrics must be equal. In the case of distinct metrics, the structure of the metrics and the manifold are strongly determined by the set, $C$, of points at which $g$ and $g^{*}$ are conformally related. The space $(M-C, g)$ is locally a warped product manifold over the hypersurface $H$. In the Lorentz setting, $C$ is empty. In the Riemannian case, $C$ contains at most two points. If $C$ is nonempty, then $H$ is isometric to a standard sphere. Furthermore, in the case that $C$ contains one point, natural hypotheses imply $M$ is diffeomorphic to $R^{n}$. If $C$ contains two points $M$ is diffeomorphic to $S^{n}$.
\end{abstract}

Introduction. This work considers the extent to which a Riemannian or Lorentz metric is determined by its geodesics. Historically, this problem has been viewed as an "inverse problem" in the calculus of variations. The problem is also related to the classical works on affine and projective connections of Cartan, Thomas, Veblen, Weyl and others.

As Hilbert and Cohn-Vossen point out in [5], this problem “...plays a certain role in modern physics because the trajectories of material points [particles] are regarded in the theory of general relativity as geodesics of a four dimensional continuum [Lorentz manifold]." To observers in a space time, only information concerning the trajectories of particles is available; the metric that determines these trajectories must be obtained through the coupling of this available information with the Einstein field equations.

An important result, which motivated the problems considered here, was obtained by Alexander in [1]. Using techniques from geometric measure theory, Alexander [1] proved the following

THEOREM. Let $d$ and $d^{*}$ be continuous distance functions on the plane such that the straight lines are the shortest paths between points for both $d$ and $d^{*}$. If $H$ is a simple closed curve bounding a region $U$ in the plane, and if for each pair of points $p, q$ in $H$, $d(p, q)=d^{*}(p, q)$, then $d$ and $d^{*}$ agree throughout $U$.

Received by the editors April 13, 1983. The contents of this paper were presented during the Special Session on Differential Geometry and Global Analysis at the 87th annual meeting of the American Mathematical Society, January 9, 1981.

1980 Mathematics Subject Classification. Primary 53A20, 53C22, 53C50.

(C) 1984 American Mathematical Society $0002-9947 / 84 \$ 1.00+\$ .25$ per page 
This result leads to the problem of differential geometry addressed by this work. Suppose that $g$ and $g^{*}$ are two metrics (either both Riemannian or both Lorentz) on a manifold $M$. Moreover, assume that $g$ and $g^{*}$ are projectively equivalent (i.e. that they have the same geodesics as point sets). Finally, suppose that they induce the same Riemannian metric on some hypersurface (codimensional-one submanifold) $H$ of $M$. What is the relationship between $g$ and $g^{*}$ ?

After recalling the classical results concerning projectively equivalent and conformally equivalent metrics, we consider the situation in which $M$ has dimension greater than two and $H$ has nondegenerate second fundamental form. Under these hypotheses we establish that unless $H$ possesses strong symmetry with respect to $M$, the two metrics agree throughout $M$.

In the situation in which the two metrics are distinct, the structure of $(M, g)$ is strongly determined by the number of conformal points in $M$, that is, those points at which $g$ and $g^{*}$ are conformally related. Under natural hypotheses the restriction of the metrics to the complement of the set of conformal points are, locally, warped product metrics. In addition, sufficient conditions are given to ensure that the restricted metrics are global warped product metrics.

If $g$ and $g^{*}$ are Lorentz metrics, then $M$ contains no conformal points. In the Riemannian setting, $M$ can have at most two conformal points. If, in fact, $M$ possesses conformal points, then $H$ is a metric sphere about each of them and is isometric to a standard sphere. If $M$ contains a single conformal point whose complement is a warped product manifold, then $M$ is diffeomorphic to $R^{n}$. If $M$ contains two conformal points, it is diffeomorphic to an $n$-sphere.

Alexander's Theorem also suggests the following: Suppose $d$ and $d^{*}$ are distance functions on a manifold $M$ that have the same distance realizing curves. Moreover, suppose that $d(p, q)=d^{*}(p, q)$ for each pair of points $p, q$ belong to a hypersurface $H$ bounding an open convex region $U$ of $M$, then $d$ and $d^{*}$ agree throughout $U$.

The author [14] has proved this assertion under the added assumption that $d$ and $d^{*}$ arise from totally convex Riemannian metrics on $M$.

The above problems have much the same flavor as the problems addressed by Muhometov [9] and Romanov [11] in their work on the "inverse kinematic problem of seismology". Briefly, this problem asks that one determine the internal structure of the earth by measuring the time required for shock waves to travel between points on the surface. Muhometov and Romanov consider this problem to be equivalent to the determination of a conformally flat, totally convex metric, $g$, on a bounded region $U$ of $R^{n}$ from knowledge of a geodesic distance function on the boundary of $U$. If such a metric exists, it is shown to be unique.

Throughout this work the word "metric" will mean either Riemannian or Lorentz metric. In statements concerning two metrics on a manifold it is implicit that both are Riemannian or both are Lorentz. All manifolds and hypersurfaces are assumed to be $C^{\infty}$ and connected. Moreover, all hypersurfaces are assumed to be smoothly imbedded. The tangent bundle to $M$ is denoted by $T M$, the tangent space to $M$ at $p$ by $M_{p}$, and the projection from $T M$ to $M$ by $\pi$. Adjectives referring to geometric structures in Lorentz manifolds follow the conventions of Sachs and $\mathrm{Wu}$ [12]. 


\section{Projectively equivalent metrics.}

1.1 Examples of projective equivalence. Two metrics on a manifold are said to be projectively equivalent if the geodesics of one are, after suitable reparametrization, the geodesics of the other. In other words, the metrics possess the same pregeodesics. Two metric manifolds $(M, g)$ and $(N, h)$ are projectively equivalent if there is a diffeomorphism $P$ from $M$ onto $N$ such that the pullback of $h$ along $P$ is projectively equivalent to $g$. The map is called a projective equivalence.

The $n$-dimensional Riemannian spaces of constant curvature are locally projectively equivalent. That is, if $(M, g)$ and $(N, h)$ are two such $n$-dimensional spaces then two points $p$ and $q$ of $M$ and $N$, respectively, have projectively equivalent neighborhoods. The projective equivalences can easily be realized by isometrically imbedding $M$ and $N$ as hypersurfaces in either $(n+1)$-dimensional Euclidean or Minkowski space and using central projections. The following example illustrates this idea.

EXAMPLE 1.1.1. Imbed $S^{n}(k)$ and $S^{n}(r)$ [the $n$-spheres of constant curvature $k$ and $r$, respectively] in $(n+1)$-dimensional Euclidean space, $E^{n+1}$, so that the two spheres intersect in an $(n-1)$-sphere which is not a great sphere of either one. Let $E^{n}$ be imbedded as the hyperplane in $E^{n+1}$ that contains the intersection of the two spheres. Using $E^{n}$, we shall construct a projective equivalence $P$ from $S^{n}(r)$ to $S^{n}(k)$ that is not a homothety. Furthermore, the pullback metric on $S^{n}(r)$ will give a second metric projectively equivalent to the standard metric, such that both induce the same metric on the $(n-1)$-sphere of intersection.

Express $S^{n}(r)$ as $H^{+}(r) \cup S^{n-1}(r) \cup H^{-}(r)$, where $S^{n-1}(r)$ is the great sphere of $S^{n}(r)$ parallel to $E^{n} ; H^{+}(r)$ is the open hemisphere that is bounded by $S^{n-1}(r)$ and does not intersect $E^{n}$; and $H^{-}(r)$ is the open hemisphere intersecting $E^{n}$. Similarly, express $S^{n}(k)$ as $H^{+}(k) \cup S^{n-1}(k) \cup H^{-}(k)$. Define a map $P$ from $S^{n}(r)$ onto $S^{n}(k)$ as follows.

(i) Let $P$ map $H^{+}(r)$ onto $H^{+}(k)$ by the composition of the central projections from $H^{+}(r)$ to $E^{n}$ through the center of $S^{n}(r)$ and from $E^{n}$ to $H^{+}(k)$ through the center of $S^{n}(k)$.

(ii) In the same way, let $P$ map $H^{-}(r)$ onto $H^{-}(k)$.

(iii) If $p$ is a point of $S^{n-1}(r)$, let $P(p)$ be that point $q$ in $S^{n-1}(k)$ such that the ray from the center of $S^{n}(r)$ through $p$ is parallel to the ray from the center of $S^{n}(k)$ through $q$.

One easily verifies that $P$ is a diffeomorphism. It is clear by construction that $P$ carries geodesics of $S^{n}(r)$ to pregeodesics of $S^{n}(k)$. Furthermore, one can see that the standard metric on $S^{n}(r)$ and the pullback metric induce the same metric on the $(n-1)$-sphere of intersection, and hence cannot be homothetically equivalent (see Figure 1.1).

By use of an intermediary hyperplane we can similarly construct local nonhomothetic projective equivalences between any pair of constantly curved spaces. Note, however, that in the case of negatively curved space $H^{n}(k)$ [ $n$-dimensional hyperbolic space of constant negative curvature $k]$, the imbedding is into $(n+1)$ dimensional Minkowski space. The center of the imbedding is the vertex of the light cone asymptotic to the imbedding. 


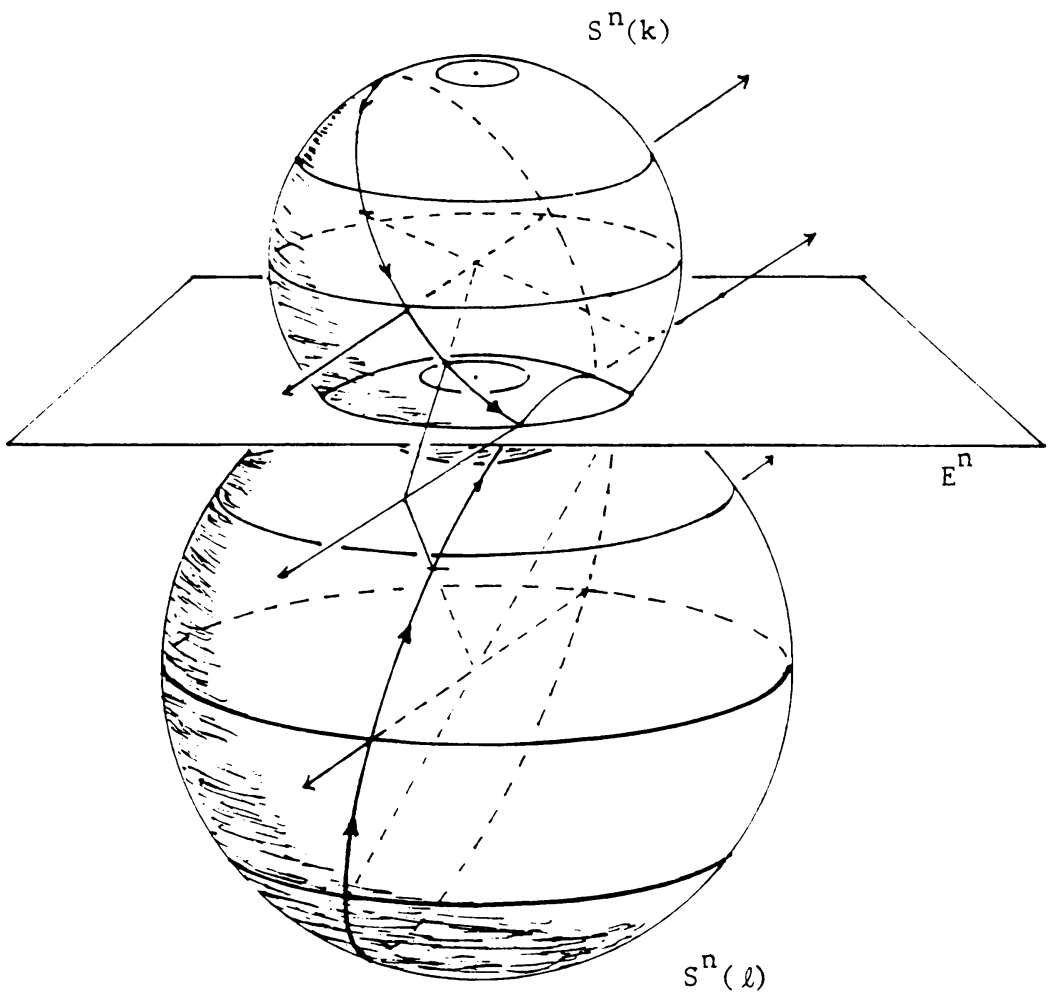

FIGURE 1.1. Construction of a nonhomothetic projective equivalence between $S^{n}(k)$ and $S^{n}(r)$.

1.2. Classical results on projectively and conformally equivalent metrics. We say that two metrics $g$ and $g^{*}$ on a manifold $M$ are conformally equivalent if there is a positive smooth function $f$ on $M$ such that $g^{*}=f g$. We say $g^{*}$ is conformally related to $g$ by $f$. The following classical result relates the connections of $g$ and $g^{*}$.

Proposition 1.2.1. Suppose $g$ and $g^{*}$ are metrics on $M$ such that $g^{*}$ is conformally related to $g$ by a function $f$. If $\nabla$ and $\nabla^{*}$ are the metric connections associated with $g$ and $g^{*}$, respectively, then for vector fields $X$ and $Y$ on $M$,

$$
\nabla_{X}^{*} Y=\nabla_{X} Y+[1 / 2 f]\{X(f) Y+Y(f) X-g(X, Y) \operatorname{grad} f\}
$$

where grad $f$ denotes the gradient of $f$ with respect to the metric $g$.

The proposition may be proved by a straightforward application of the Koszul formula,

$$
\begin{aligned}
g\left(\nabla_{X} Y, Z\right)=\frac{1}{2}\{ & X g(Y, Z)+Y g(X, Z)-Z g(X, Y) \\
& -g(X,[Y, Z])-g(Y,[X, Z])+g(Z,[X, Y])\} .
\end{aligned}
$$

For example, see the paper [8] of Kulkarni. 
Just as we can state the relationship between the connections of conformally related metrics on a manifold $M$, we can give the relationship between projectively equivalent, torsion-free connections $\nabla^{*}$ and $\nabla$ on a manifold. The following fundamental proposition is due to Weyl [15]. A proof of statement (i) may be found in Spivak's text [13]. The proof of (ii) is given in Eisenhart's book [4].

Proposition 1.2. Let $M$ be an n-dimensional manifold with torsion-free connections $\nabla$ and $\nabla^{*}$.

(i) The connections are projectively equivalent if and only if there is a unique one-form $\omega$ such that for all vector fields $X$ and $Y$ on $M, \nabla_{X}^{*} Y=\nabla_{X} Y+\omega(X) Y+$ $\omega(Y) X$.

(ii) If $\nabla$ and $\nabla^{*}$ are projectively equivalent metric connections corresponding to the metrics $g$ and $g^{*}$, respectively, then the one-form $\omega$ of (i) is exact. Moreover, $\omega=d \ln (Q)$ where $Q$ is the function such that $d V^{*}=Q^{n+1} d V$, and $d V$ and $d V^{*}$ are the canonical volume elements associated with $g$ and $g^{*}$, respectively. [In coordinate notation, $Q^{n+1}=\sqrt{\left|\operatorname{det} g^{*}\right| /|\operatorname{det} g|}$.]

As an immediate consequence of (ii) above, we see that if $\nabla$ and $\nabla^{*}$ are projectively equivalent metric connections, and $X$ and $Y$ are vector fields on $M$, then $\nabla_{X}^{*} Y=\nabla_{X} Y+X(\ln Q) Y+Y(\ln Q) X$. Henceforth, the above equation shall be referred to as the Weyl formula.

Propositions 1.2.1 and 1.2.2 provide an easy proof of the following classical result. This theorem will be an important tool in establishing that, under suitable hypotheses, a metric is determined by its geodesics and the metric it induces along a hypersurface.

THEOREM 1.2.3. If the metrics $g$ and $g^{*}$ on the manifold $M$ are projectively and conformally equivalent and $M$ has dimension two or more, then $g$ and $g^{*}$ are homothetically related.

Proof. Let $g^{*}$ be conformally related to $g$ by the function $f$. Given any pair of vector fields $X$ and $Y$ on $M$ we can compute the difference, $\nabla_{X}^{*} Y-\nabla_{X} Y$, by using either Proposition 1.2.1 or 1.2.2. When the results of these two computations are equated we see that all vector fields $X$ and $Y$ on $M$ must satisfy the equation

$$
\omega(X) Y+\omega(Y) X=[1 /(2 f)]\{d f(X) Y+d f(Y) X-g(X, Y) \operatorname{grad} f\} \text {. }
$$

Clearly, this equation can be satisfied for all $X$ and $Y$ only if $\operatorname{grad} f$ is identically zero on $\mathrm{M}$. Thus $f$ must be constant on $M$ and consequently $g$ and $g^{*}$ are homothetically related. Q.E.D.

1.3. Warped product examples. An important class of metric manifolds, for which one can construct projectively equivalent metrics inducing the same Riemannian metric on a hypersurface, are the codimension-one warped product manifolds.

Definition 1.3.1. Let $(M, g)$ and $(N, h)$ be Riemannian manifolds and let $f$ be a smooth, positive, real valued function on $N$. The Riemannian warped product of $M$ and $N$ through $f$ is the manifold $M \times N$ together with the metric, $f^{2} g+h$, defined as follows: If $\left(X_{i}, Y_{i}\right)$ belongs to $(M \times N)_{(p, q)}$, for $i \in\{1,2\}$, then

$$
\left(f^{2} g+h\right)_{(p, q)}\left[\left(X_{1}, Y_{1}\right),\left(X_{2}, Y_{2}\right)\right]=f^{2}(q) g\left(X_{1}, X_{2}\right)+h\left(Y_{1}, Y_{2}\right) \text {. }
$$


The semi-Riemannian warped product of $M$ with $N$ through $f$ is the manifold $M \times N$ together with the semi-Riemannian metric $f^{2} g-h$. If the dimension of $N$ is one, we shall call the semi-Riemannian warped product a Lorentz warped product. These manifolds will be denoted by $\Re\left(M \times_{f} N\right)$ in the Riemannian case and $\mathscr{L}\left(M \times_{f} N\right)$ in the semi-Riemannian case. When it is unnecessary to distinguish between the two cases, we shall simply write $M \times_{f} N$. The submanifolds $M \times\{q\}$ will be called leaves, and the submanifolds $\{p\} \times N$, fibers of the warped product. Finally, the dimension of $N$ will be referred to as the codimension of the warped product.

We shall investigate the projective properties of the codimension-one warped product manifolds in this section. Let $(H, h)$ be a Riemannian manifold with metric connection $D$, and let $(I, m)$ be a one-dimensional, connected Riemannian manifold. Finally, let $\alpha$ be a positive, smooth function on $I$ and consider the warped product $(H, h) \times_{\alpha}(I, m)$. (There is no need to distinguish between the Lorentz or Riemannian cases in the present context.)

If $E$ is a vector tangent to $H \times I$ at $(p, u)$, then $E$ can be expressed uniquely as $\mathcal{H}(E)+\mathscr{K}(E)$, where $\mathcal{H}(E)$ is tangent to the leaf $H \times\{u\}$, and $\mathscr{N}(E)$ is tangent to the fiber $\{p\} \times I$ at $(p, u)$.

The second fundamental form of the leaves, which measures the difference between the connection $\nabla$ of $(H, h) \times_{\alpha}(I, m)$ and the connection $D$ of the fibers, may be used to obtain a $(1,2)$ tensor $T$ on $(H, h) \times_{\alpha}(I, m)$. As in O'Neill's paper [10], we define $T$ by

$$
T_{E} F=\mathscr{N}\left[\nabla_{\mathcal{K}(E)} \mathcal{H}(F)\right]+\mathcal{H}\left[\nabla_{\mathcal{K}(E)} \mathfrak{M}(F)\right]
$$

for any vector fields $E$ and $F$ on $H \times I$.

Vector fields on $H$ and $I$ have canonical extensions to the warped product manifold $(H, h) \times_{\alpha}(I, m)$. More explicitly, we identify the vector field $X$ on $H$ (or $I)$ with the vector field $(X, 0)($ or $(0, X))$ on $H \times I$. (Here 0 denotes the zero vector field on $H$ or $I$, depending upon context.) These canonical extensions of the vector fields on $H$ and $I$ are called the basic vector fields on $(H, h) \times_{\alpha}(I, m)$.

To keep notation manageable, whenever $X$ denotes a vector field on $H$ or $I$, we shall also let $X$ stand for the corresponding basic vector field on the warped product $(H, h) \times_{\alpha}(I, m)$. In addition to maintaining manageable notation, this convention facilitates the comparison of covariant derivatives of basic vector fields with their counterparts on $H$ and $I$. For example, suppose $X$ and $Y$ are vector fields on $I$. A straightforward application of the Koszul formula shows that $\nabla_{X} Y$, the covariant derivative of the basic vector field $Y$ with respect to the basic vector field $X$ on $H \times I$, is the basic vector field corresponding to the covariant derivative of $Y$ with respect to $X$ on $I$. Thus we say $\nabla_{X} Y$ is the same on both $(I, m)$ and $(H, h) \times_{\alpha}(I, m)$. Further application of the Koszul formula yields the following lemma due to Bishop and O'Neill [3].

LEMMA 1.3.2. Let $X$ and $Y$ be vector fields on $I$, and $V$ and $W$ vector fields on $H$. Then

(i) $\nabla_{X} Y$ is the same on either I or $(H, h) \times_{\alpha}(I, m)$,

(ii) $\nabla_{X} V=\nabla_{V} X=T_{V} X=(X \alpha / \alpha) V$, 
(iii) $\mathfrak{N}\left(\nabla_{V} W\right)=T_{V} W=-\alpha h(V, W) \operatorname{grad} \alpha$,

(iv) $\mathcal{H}\left(\nabla_{V} W\right)=D_{V} W$.

The examples of projectively equivalent metrics constructed in subsection 1.1 are, in fact, special cases of completed warped products of an $(n-1)$-sphere with an interval of $E^{1}$. The above lemma enables us to generalize these constructions. Moreover, as we shall see in $\S 3$, the following construction is essentially the only possible construction of projectively equivalent metrics inducing the same Riemannian metric on a hypersurface.

Proposition 1.3.3. Suppose that $m$ and $m^{*}$ are metrics on $I$, and that $\alpha$ and $\beta$ are positive smooth functions on I. The warped product manifolds $(H, h) \times_{\alpha}(I, m)$ and $(H, h) \times_{\beta}\left(I, m^{*}\right)$ have the same pregeodesics if and only if there exist constants $c$ and $k$ such that

$$
\beta^{2}=c^{2} \alpha^{2} /\left(1+k \alpha^{2}\right),
$$

and

$$
m^{*}=\left[c^{2} /\left(1+k \alpha^{2}\right)^{2}\right] m .
$$

Proof. Let $g$ and $g^{*}$ denote the metrics of $(H, h) \times_{\alpha}(I, m)$ and $(H, h) \times_{\beta}\left(I, m^{*}\right)$, respectively. Let $\nabla$ and $\nabla^{*}$ be the corresponding metric connections. Finally, since $I$ is one dimensional, $m$ and $m^{*}$ are conformally related. Let $m^{*}=f m$.

By Proposition 1.2.2, the two metrics $g$ and $g^{*}$ are projectively equivalent if and only if there is an exact one-form $\omega$ such that

$$
\nabla_{E}^{*} F-\nabla_{E} F=\omega(E) F+\omega(F) E
$$

for any vector fields $E$ and $F$ on $H \times I$.

Since the difference of two connections is a $(1,2)$ tensor, the value of $\nabla_{E}^{*} F-\nabla_{E} F$ at a point $p$ is determined by $E_{p}$ and $F_{p}$. We may choose any convenient extension of $E_{p}$ and $F_{p}$ to evaluate this difference. Thus we take $E$ and $F$ to be $X+V$ and $Y+W$, respectively, where $X$ and $Y$ are vector fields on $I$, and $V$ and $W$ are vector fields on $H$. By Lemma 1.3.2 we have

$$
\begin{aligned}
\nabla_{E}^{*} F-\nabla_{E} F= & \nabla_{X}^{*} Y-\nabla_{X} Y+[(X \beta / \beta)-(X \alpha / \alpha)] W \\
& +[(Y \beta / \beta)-(Y \alpha / \alpha) V] \\
& +h(V, W)\left[\alpha \operatorname{grad} \alpha-\beta \operatorname{grad}^{*} \beta\right]
\end{aligned}
$$

where grad and grad* denote the gradients with respect to $g$ and $g^{*}$.

From (i) of Lemma 1.3.2 we know that $\nabla_{X}^{*} Y$ and $\nabla_{X} Y$ can be computed on $\left(I, m^{*}\right)$ and $(I, m)$. Moreover, since $I$ is one dimensional the metrics $m^{*}$ and $m$ are necessarily projectively equivalent. Thus the Weyl formula yields

$$
\nabla_{X}^{*} Y-\nabla_{X} Y=X(\ln \sqrt[4]{f}) Y+Y(\ln \sqrt[4]{f}) X
$$


Substituting this result into (2) and simplifying we have

$$
\begin{aligned}
\nabla_{E}^{*} F-\nabla_{E} F= & X(\ln \sqrt[4]{f}) Y+Y(\ln \sqrt[4]{f}) X \\
& +X(\ln (\beta / \alpha)) W+Y(\ln (\beta / \alpha)) V \\
& +h(V, W)\left[\alpha \operatorname{grad} \alpha-\beta \operatorname{grad}^{*} \beta\right] .
\end{aligned}
$$

Furthermore, since $V$ and $W$ are vector fields on $H$, equation (4) may be expressed as

$$
\begin{aligned}
\nabla_{E}^{*} F-\nabla_{E} F= & E(\ln \sqrt[4]{f}) Y+E(\ln \beta / \alpha) W \\
& +F(\ln \sqrt[4]{f}) X+F(\ln \beta / \alpha) V \\
& +h(V, W)\left[\alpha \operatorname{grad} \alpha-\beta \operatorname{grad}^{*} \beta\right]
\end{aligned}
$$

From equation (5) it is apparent that the difference, $\nabla_{E}^{*} F-\nabla_{E} F$, can be expressed as in (1) if and only if $\alpha, \beta$ and $f$ satisfy

$$
\alpha \operatorname{grad} \alpha-\beta \operatorname{grad}^{*} \beta=0
$$

and

$$
f=\beta^{4} / c^{2} \alpha^{4}
$$

for some nonzero constant $c$. Expressing $\operatorname{grad} \alpha$ and $\operatorname{grad}^{*} \beta$ in terms of an $m$-unit vector field $N$ on $I$ and combining with equation (7) yields

$$
\frac{1}{\alpha^{3}} N(\alpha)=\frac{c^{2}}{\beta^{3}} N(\beta) \text {. }
$$

Thus (i) and (ii) follows from (7) and (8).

It is an easy matter to show that if $f$ and $m^{*}$ satisfy (i) and (ii) then equations (7) and (8) are satisfied and hence $g$ and $g^{*}$ are projectively equivalent. Q.E.D.

From the above proposition it is clear that we can construct a one-parameter family of projectively equivalent metrics on $H \times I$, all of whose members induce the same metric on some leaf $H \times\{u\}$.

2. Projectively equivalent metrics inducing the same metric on a hypersurface.

2.1. Recovery of $g^{*}$. In this section we shall determine conditions sufficient to insure that projectively equivalent metrics, inducing the same Riemannian metric along a hypersurface, are identically equal. The following proposition will be an important tool in this and the following section.

Proposition 2.1.1. Let $g$ and $g^{*}$ be projectively equivalent metrics on a manifold $M$, and let $\nabla$ and $\nabla^{*}$ be the corresponding metric connections. Choose a geodesic $\gamma$ of the metric $g$ and let $V$ be its velocity. If $X$ and $Y$ are parallel vector fields along $\gamma$ with 
respect to the connection $\nabla$, then

$$
\begin{aligned}
g^{*}(X(t), Y(t))=\frac{Q^{2}(t)}{Q^{2}(0)} g^{*}( & {\left[\frac{\int_{0}^{t} X(Q)(s) d s}{Q(0)}\right] V(0)+X(0), } \\
& {\left.\left[\frac{\int_{0}^{t} Y(Q)(s) d s}{Q(0)}\right] V(0)+Y(0)\right) }
\end{aligned}
$$

where $Q(t)$ denotes $Q \circ \gamma(t)$.

Proof. The proof consists of solving a sequence of differential equations. We begin by differentiating $g^{*}(V, V)$. Recalling the Weyl formula, we obtain

$$
\frac{d}{d t} g^{*}(V, V)=2 g^{*}\left(\nabla_{V}^{*} V, V\right)=2 g^{*}\left(\nabla_{V}^{*} V+2 V(\ln Q) V, V\right) \text {. }
$$

Since $\gamma$ is a geodesic with respect to $g$, we know that $\nabla_{V}^{*} V=0$. Thus $g^{*}(V, V)$ must satisfy the differential equation

$$
\frac{d}{d t} g^{*}(V(t), V(t))=4 \frac{d}{d t}[\ln Q(t)] g^{*}(V(t), V(t)) .
$$

Hence

$$
g^{*}(V(t), V(t))=\left[Q^{4}(t) / Q^{4}(0)\right] g^{*}(V(0), V(0))
$$

Using this result, we can compute $g^{*}(V(t), X(t))$. Differentiating, applying the Weyl formula and keeping in mind that both $V$ and $X$ are parallel along $\gamma$ with respect to $\nabla$, we have

$$
\frac{d}{d t} g^{*}(V, X)=3 V(\ln Q) g^{*}(V, X)+X(\ln Q) g^{*}(V, V) .
$$

Applying the result of (1) above, we see that $g^{*}(V, X)$ must satisfy the differential equation

$$
\begin{aligned}
\frac{d}{d t} g^{*}(V(t), X(t))= & 3 \frac{d}{d t}[\ln Q(t)] g^{*}(V(t), X(t)) \\
& +X(\ln Q)(t) \frac{Q^{4}(t)}{Q^{4}(0)} g^{*}(V(0), V(0))
\end{aligned}
$$

By assuming a solution of the form $c(t) e^{a(t)}$, and solving for $c(t)$ and $a(t)$, we obtain

$$
\begin{aligned}
g^{*}(V(t), X(t))= & {\left[Q^{3}(t) / Q^{4}(0)\right]\left[\int_{0}^{t} X(Q)(s) d s\right] g^{*}(V(0), V(0)) } \\
& +\left[Q^{3}(t) / Q^{3}(0)\right] g^{*}(V(0), X(0))
\end{aligned}
$$

Finally, using the result of (2) and proceeding as before we find that $g^{*}(X, X)$ must satisfy the differential equation

$$
\begin{aligned}
\frac{d}{d t} g^{*}(X(t), X(t))= & 2 \frac{d}{d t}[\ln Q(t)] g^{*}(X(t), X(t))+\left[2 X(\ln Q) Q^{3}(t) / Q^{3}(0)\right] \\
& \times\left\{\left[\frac{\int_{0}^{t} X(Q)(s) d s}{Q(0)}\right] g^{*}(V(0), X(0))+g^{*}(V(0), V(0))\right\} .
\end{aligned}
$$


Solving as above gives

$$
\begin{aligned}
& g^{*}(X(t), X(t))=\frac{Q^{2}(t)}{Q^{2}(0)} g^{*}\left(\left[\frac{\int_{0}^{t} X(Q)(s) d s}{Q(0)}\right] V(0)+X(0)\right. \\
& {\left.\left[\frac{\int_{0}^{i} X(Q)(s) d s}{Q(0)}\right] V(0)+X(0)\right) . }
\end{aligned}
$$

Polarization now yields the desired result. Q.E.D.

2.2. Aligned metrics. We now begin the investigation of our central problem. Suppose that $g$ and $g^{*}$ are projectively equivalent metrics on a connected manifold $M$ and that they induce the same Riemannian metric on some hypersurface $H$. What is the relationship between $g$ and $g^{*}$ ?

As in the preceding subsection, we let $\nabla$ and $\nabla^{*}$ denote the metric connections corresponding to $g$ and $g^{*}$. Locally on $H$, we may choose unit normal vector fields with respect to $g$ and $g^{*}$; call these normal fields $N$ and $N^{*}$. Finally, we let $S$ and $S^{*}$ be the second fundamental forms of $H$ with respect to $g$ and $g^{*}$ (in other words, for any vector fields $X$ and $Y$ tangent to $H$, the vector field $S(X, Y)$ is the component of $\nabla_{X}^{*} Y$ g-normal to $H$ ).

Definition 2.2.1. A hypersurface of a metric manifold $(M, g)$ is said to be properly curved in $(M, g)$ if its second fundamental form is nondegenerate on a dense subset of the given hypersurface.

Definition 2.2.2. Let $g$ and $g^{*}$ be metrics on a manifold $M$. The metrics are said to be aligned along a hypersurface $H$ if (i) they induce the same Riemannian metric on $H$, and (ii) $g$ and $g^{*}$ have the same normal lines along $H$.

While the notion of aligned metrics along a hypersurface seems quite strong, the following theorem shows that the idea occurs naturally in our context.

THEOREM 2.2.3. Let $g$ and $g^{*}$ be projectively equivalent metrics on the manifold $M$ that induce the same Riemannian metric along a hypersurface $H$ of $M$. If $H$ is properly curved in $(M, g)$ and $M$ has dimension greater than two, then

(i) $g$ and $g^{*}$ are aligned along $H$,

(ii) $Q$ is constant along $H$,

(iii) there is a positive constant, $c$, such that if $N$ and $N^{*}$ are any pair of local unit normal fields on $H$ pointing in the same direction, then $N^{*}=c N$.

Proof. Since $g$ and $g^{*}$ induce the same metric on $H$, the projection of their connections to $H$ must agree. Thus if $N$ and $N^{*}$ are local unit normals to $H$, and $X$ and $Y$ are vector fields tangent to $H$,

$$
\nabla_{X}^{*} Y-\left[g^{*}\left(\nabla_{X}^{*} Y, N^{*}\right) / g^{*}\left(N^{*}, N^{*}\right)\right] N^{*}=\nabla_{X} Y-\left[g\left(\nabla_{X} Y, N\right) / g(N, N)\right] N \text {. }
$$

Applying the Weyl formula to this expression, we obtain

$$
\begin{aligned}
\nabla_{X} Y= & X(\ln Q) Y+Y(\ln Q) X \\
& +\left[g^{*}\left(\nabla_{X} Y+X(\ln Q) Y+Y(\ln Q) X, N^{*}\right) / g^{*}\left(N^{*}, N^{*}\right)\right] N^{*} \\
= & \nabla_{X} Y-\left[g\left(\nabla_{X} Y, N\right) / g(N, N)\right] N .
\end{aligned}
$$

Since $X$ and $Y$ are $g^{*}$-orthogonal to $N^{*}$, the above equation can be simplified. 
Noting that $g^{*}\left(N^{*}, N^{*}\right)=g(N, N)= \pm 1$, we have

$$
X(\ln Q) Y+Y(\ln Q) X=g(N, N)\left[g^{*}\left(\nabla_{X} Y, N^{*}\right) N^{*}-g\left(\nabla_{X} Y, N\right) N\right] .
$$

Since any vector $V$ tangent to $M$ at a point of $H$ can be expressed as

$$
V=\{V-[g(V, N) / g(N, N)] N\}+[g(V, N) / g(N, N)] N,
$$

it follows that

$$
g^{*}\left(V, N^{*}\right)=g(V, N) g^{*}\left(N, N^{*}\right) / g(N, N) .
$$

Combining (1) and (2), we see that for the particular case when $X$ and $Y$ are equal,

$$
2 X(\ln Q) X=g\left(\nabla_{X} X, N\right)\left[g^{*}\left(N, N^{*}\right) N^{*}-g(N, N) N\right] \text {. }
$$

If $Y$ is tangent to $H$ and orthogonal to $X$, it follows that

$$
0=g\left(\nabla_{X} X, N\right) g^{*}\left(N, N^{*}\right) g\left(N^{*}, Y\right) \text {. }
$$

By definition, $S(X, X)=g(N, N) g\left(\nabla_{X} X, N\right) N$. Furthermore, at a point $p$ in $H$ where $S$ is nondegenerate, the set $U_{p}$ of vectors $X$ which are tangent to $H$ at $p$ and for which $S(X, X)$ is nonzero is dense in $H_{p}$. Thus, the collection of vectors $Y$, which are orthogonal to some vector of $U_{p}$ is dense in $H_{p}$. Hence it follows from equation (4) that at those points of $H$ for which the second fundamental form $S$ is nondegenerate, the vector $N^{*}$ must also be $g$-orthogonal to $H$. Since $H$ is properly curved, the metrics $g$ and $g^{*}$ have the same normal lines along $H$ and hence are aligned along $H$. Applying this result to equation (3), we see that $2 X(\ln Q) g(X, X)$ $=0$ for any vector $X$ tangent to $H$. Therefore $X(Q)=0$ and thus $Q$ must be constant on $H$.

Finally, recall that when $g$ and $g^{*}$ are expressed in local coordinates, $Q^{(2 n+1)}=$ $\left|\operatorname{det} g^{*}\right| /|\operatorname{det} g|$. Since $Q$ is constant on $H$ and the metrics $g$ and $g^{*}$ are aligned along $H$, it follows that there exists a fixed positive constant $c$ such that whenever $N$ and $N^{*}$ are local unit normals on $H$ pointing in the same direction, they must satisfy $N^{*}=c N$. Moreover, the value of $c$ is $[1 / Q(H)]^{n+1}$ (where $Q(H)$ is the value of $Q$ at any point of $H$ ). Q.E.D.

REMARK. In the proof of the above theorem, we did not invoke the hypotheses concerning the curvature of $H$ and the dimension of $M$ until after equation (4) had been established. These hypotheses were necessary to show that the normal lines of $H$ with respect to the two metrics were collinear. Once this fact had been established, the rest of the theorem followed easily. These observations lead to the following

COROLlaRY 2.2.4. If $g$ and $g^{*}$ are projectively equivalent metrics on $M$ and are aligned along the hypersurface $H$, then (i) $Q$ is constant on $H$ and (ii) there is a constant c such that if $N$ and $N^{*}$ are any local unit normals on $H$ pointing in the same direction, then $N^{*}=c N$.

REMARK. In Theorem 2.2.3, the hypothesis that $M$ have dimension at least three cannot be weakened. Examples of two-dimensional, projectively equivalent metric manifolds exist such that the two metrics in question induce the same metric on a properly curved hypersurface (i.e. a curve). Yet the metrics are not aligned along this curve [14]. 
REMARK. If, in Theorem 2.2.3, the hypothesis that $H$ is properly curved is deleted, then $g$ and $g^{*}$ need not be aligned. For example, let $x^{1}, \ldots, x^{n}$ be the usual coordinate functions on $R^{n}$. Let $g, g^{*}$ be the metrics

$$
g=\sum_{i=1}^{n} d x^{i} \otimes d x^{i}, \quad g^{*}=\sum_{i, j=1}^{n} g_{i j}^{*} d x^{i} \otimes d x^{j}
$$

where the $g_{i j}^{*}$ are constants satisfying

(i) $g_{i j}^{*}=\delta_{i j}$ for $i, j \leqslant n-1$;

(ii) $g_{n n}^{*}=1, g_{i n}^{*}=g_{n i}^{*}$ for all $i$ less than $n$;

(iii) $0<1-\sum_{i=1}^{n-1} g_{i n}^{* 2}<1$.

The metrics $g$ and $g^{*}$ are Riemannian, projectively equivalent, and induce the same metric on the totally geodesic hypersurface $x^{n}=0$; yet they are not aligned along this hypersurface.

2.3. Equireflective hypersurfaces. The examples of $\$ 1$ demonstrate the existence of distinct projectively equivalent metrics on a manifold $M$ that induce the same Riemannian metric on some hypersurface of the manifold. However, in each of the examples cited, the hypersurface chosen possesses a great deal of symmetry with respect to its ambient manifold. In this subsection we shall demonstrate the necessity of such symmetry.

DEFINITION 2.3.1. Let $H$ be a Riemannian hypersurface of the metric manifold $(M, g)$. Suppose that $\gamma$ is a geodesic segment in $M$ with velocity $V$, and that the endpoints, $p$ and $q$, of $\gamma$ lie in $H$. Let $N_{p}$ and $N_{q}$ be the unit normals to $H$ at $p$ and $q$. The geodesic $\gamma$ is said to be equireflective with respect to its endpoints if

$$
\left|g\left(N_{p}, V\right)\right|=\left|g\left(N_{q}, V\right)\right| \text {. }
$$

We say that $H$ is equireflective if each geodesic that joins two points of $H$ is equireflective with respect to those points.

REMARK. As will soon be evident, the leaves of a codimension-one warped product manifold are equireflective. Thus any Riemannian manifold can be imbedded as an equireflective hypersurface of some Lorentz or Riemannian manifold.

In the Riemannian setting, we can interpet the above definition as follows: A hypersurface $H$ of a Riemannian manifold $(M, g)$ is equireflective if for each geodesic, $\gamma$, in $M$ that joins two points, $p$ and $q$, of $H$, the angle between $\gamma$ and the normal line to $H$ at $p$ is equal to the angle between $\gamma$ and thenormal line to $H$ at $q$.

In the Lorentz setting recall that at any point $p$ of $H$, there is an instantaneous observer $\left(p, Z_{p}\right)$ whose instantaneous rest space is $H_{p}$. Let $\left(p, Z_{p}\right)$ and $\left(q, Z_{q}\right)$ be any pair of these instantaneous observers on $H$ that are joined by a geodesic $\gamma$ in $M$. $H$ is equireflective if and only if for each such pair the energy of $\gamma$ as observed by $\left(p, Z_{p}\right)$ is equal to the energy of $\gamma$ as observed by $\left(q, Z_{q}\right)$.

Having established the idea of an equireflective hypersurface, we are ready to state one of our main results.

THEOREM 2.3.2. Let $g$ and $g^{*}$ be projectively equivalent metrics on the manifold $M$, that are aligned along the hypersurface. If $H$ is not equireflective in $(M, g)$, then $g$ and $g^{*}$ are equal on $M$. 
As an immediate consequence of Theorems 2.3.2 and 2.2.3, we have the following

COROLlaRY 2.3.3. Suppose that $g$ and $g^{*}$ are projectively equivalent metrics on the manifold $M$ and that they induce the same Riemannian metric on a hypersurface $H$. If $M$ has dimension greater than two, and $H$ is properly curved and not equireflective in $(M, g)$, then $g$ and $g^{*}$ agree throughout $M$.

Proof of Theorem 2.3.2. We first show that $g$ and $g^{*}$ are equal on $H$.

Let $\gamma$ be a geodesic in $M$, with velocity $V$, which joins $H$ at $p$ and $q$, and is not equireflective with respect to $p$ and $q$. Let $N$ and $N^{*}$ denote unit normal fields at either $p$ or $q$, and assume that $N$ and $N^{*}$ point in the same direction.

At $p$ and $q$, the velocity of $\gamma$ can be expressed as

$$
V_{p}=e_{p} N_{p}+h_{p} T_{p}, \quad V_{q}=e_{q} N_{q}+h_{q} T_{q}
$$

where $T_{p}$ and $T_{q}$ are unit tangents to $H$ at $p$ and $q$, respectively. Since $\gamma$ is a geodesic, we have

$$
e_{p}^{2} g(N, N)+h_{p}^{2}=e_{q}^{2} g(N, N)+h_{q}^{2} .
$$

By Proposition 2.1.1, we know that

$$
g^{*}\left(V_{q}, V_{q}\right)=\left[Q^{4}(q) / Q^{4}(p)\right] g^{*}\left(V_{p}, V_{p}\right)
$$

However, by Corollary 2.2.4, $Q$ is constant on $H$ and there is a positive constant $c$ such that $N^{*}=c N$. Thus equations (1) and (3) yield

$$
e_{p}^{2} g(N, N) / c^{2}+h_{p}^{2}=e_{q}^{2} g(N, N) / c^{2}+h_{q}^{2} .
$$

Equations (2) and (4) can be satisfied simultaneously only if $e_{p}^{2}=e_{q}^{2}$ or $c^{2}=1$. However, the nonequireflective choice of $\gamma$ rules out the case $e_{q}^{2}=e_{p}^{2}$. Thus $c=1$, and $g$ and $g^{*}$ are equal on $H$.

To show that $g$ and $g^{*}$ are equal at each point of $M$, we now need only show that they are conformally related on $M$.

For a point $p$ in $H$, consider the open neighborhood $U$ of $(p, 0)$ in $T M$ such that the restriction of the exponential map of $g$ to each fiber is a diffeomorphism onto $\pi(U)$. The set, $\mathcal{G}$, of points in $\pi(U)$ that can be joined to $\pi(U) \cap H$ by a geodesic in $\pi(U)$, transverse to $H$, forms an open neighborhood of $\pi(U) \cap H$. If $x$ is in $\mathcal{G}$ but not in $H$, the inverse image, $D_{x}$, of $\pi(U) \cap H$ under the exponential map is a hypersurface in the tangent space $M_{x}$. Furthermore, $D_{x}$ does not lie in any vector subspace of $M_{x}$. Consequently, the values of $g^{*}(V, V)$ for $V$ in $M_{x}$ are determined by the values that this quadratic form assumes on $D_{x}$.

Consider any vector $\hat{v}$ in $D_{x}$ and let $\gamma$ be the geodesic with respect to $g$ having velocity $V$ such that $V(0)=\hat{v}$. By Proposition 2.1.1, we know that

$$
g^{*}(V(1), V(1))=\left[Q^{4} \circ \gamma(1) / Q^{4}(x)\right] g^{*}(V(0), V(0)) .
$$

However, by definition, $\gamma(1)$ belongs to $H$. Thus, from the fact that $g$ and $g^{*}$ are equal on $H$, we have

$$
g^{*}(V(1), V(1))=g(V(1), V(1))=g(\hat{v}, \hat{v}) .
$$


This fact together with the fact that $Q$ is identically one on $H$ yield $g^{*}(\hat{v}, \hat{v})=$ $Q^{4}(x) g(\hat{v}, \hat{v})$ for all $\hat{v}$ in $D_{x}$. Consequently, $g^{*}$ is conformally related to $g$ at $x$ and hence $g$ and $g^{*}$ are conformally related throughout $\mathcal{G}$. Thus by Theorem 1.2.3, $g$ and $g^{*}$ are equal on $\mathcal{G}$. Now let $B$ be the maximal open subset of $M$ on which $g$ and $g^{*}$ are equal. By applying the same argument as above, one can show that $B$ must coincide with $M$. Q.E.D.

The last half of the proof above also yields the following

COROLlaRY 2.3.4. If $g$ and $g^{*}$ are projectively equivalent metrics that are equal along some hypersurface $H$ of $M$, then $g$ and $g^{*}$ are equal on $M$.

3. The structure of distinct aligned metrics. Throughout this section we assume that $g$ and $g^{*}$ are distinct projectively equivalent metrics on a manifold $M$ and that they are aligned along some hypersurface $H$. Recall that $g$ and $g^{*}$ are aligned along $H$ if (i) they induce the same Riemannian metric on $H$, and (ii) they have the same normal lines along $H$. By Theorem 2.2.3, the second condition follows from the first if $M$ has dimension greater than two, and the second fundamental form of $H$ is nondegenerate.

In addition to assuming that $g$ and $g^{*}$ are aligned on $H$, we asume that we can make a global choice of unit normal field on $H$. As before, $N$ and $N^{*}$ will denote the $g$ - and $\mathrm{g}^{*}$-unit normal fields. We shall always assume that $N$ and $N^{*}$ are chosen so as to point in the same direction. Moreover, we assume that $g$ and $g^{*}$ have been appropriately labelled so that the constant $c$, for which $N^{*}=c N$, is positive and less than one. Finally, we take $\nabla$ and $\nabla^{*}$ to be the connections of $g$ and $g^{*}$, respectively.

In order to investigate the full relationship between $g$ and $g^{*}$, we need to develop a means of comparing them away from the hypersurface. This comparison will be accomplished by using $g$ to view $g^{*}$ as a $(1,1)$ tensor on $M$. We shall show that the warped product examples of $\S 1$ are essentially the only examples of projectively equivalent aligned metrics.

Where possible, we shall simultaneously treat both the Lorentz and Riemannian cases. Occasionally, however, we shall need to treat the two settings separately.

Finally, although we shall be dealing with two metrics on $M$, metric notions, such as unit speed, orthogonality, exponential map, lightlike and so on, should be assumed to be in reference to the metric $g$ unless stated otherwise.

3.1. Definition of the maximal line field. The metric $g^{*}$ determines, on each tangent space to $M$, a symmetric linear operator $G$, where $G_{p}(v)$ is that unique vector in $M_{p}$ such that $g\left(G_{p}(v), w\right)=g^{*}(v, w)$ for all $w$ in $M_{p}$. Since $G$ is symmetric and nondegenerate, it is diagonalizable. Furthermore, the invariant subspaces corresponding to distinct eigenvalues are mutually orthogonal with respect to both $g$ and $g^{*}$.

The nondegeneracy of $G$ guarantees that all of its eigenvalues are positive. While this fact is obvious in the Riemannian setting, some comment is called for in the Lorentz setting. Let $v_{1}, \ldots, v_{n}$ be an orthonormal basis of eigenvectors of $G$ on $M_{p}$, for some $p$ in $H$. Let $\lambda_{1}(p), \ldots, \lambda_{n}(p)$ be their associated eigenvalues. Since $g$ and $g^{*}$ are aligned, it is clear that we may set $v_{1}=N$, and take $v_{2}, \ldots, v_{n}$ to be tangent to $H$. 
Since $v_{2}, \ldots, v_{n}$ are spacelike with respect to both metrics, $N$ is timelike with respect to both. Furthermore, since $g\left(G\left(v_{i}\right), v_{j}\right)=\lambda_{i}(p) g\left(v_{i}, v_{j}\right)=g^{*}\left(v_{i}, v_{j}\right)$, it follows in the Lorentz setting, as in the Riemannian setting, that the eigenvalues of $G$ along $H$ are $1 / c^{2}$ and 1 . It is well known that there are $n$ continuous functions $\lambda_{1}, \ldots, \lambda_{n}$ on $M$ that represent the eigenvalues of $G$ on $M[6, \mathrm{p} .122]$. Since these functions are positive along $H$ and nowhere zero on $M$, they must be positive on $M$.

From the preceding paragraph it follows that $G$ has a simple maximum eigenvalue at each point of $H$ in both the Riemannian and Lorentz settings. We let $\mathscr{Q}$ be the connected open subset of $M$ containing $H$ on which $G$ has a simple maximum eigenvalue. The maximum eigenvalue is a smooth function on $\mathscr{D}$. Furthermore, the line field $\mathfrak{T}$, formed by the eigenspaces of the maximal eigenvalues, is smooth on $\mathbb{D}$ [6]. We shall call $\mathfrak{R}$ the maximal line field on $M$.

The line field $\mathfrak{N}$ has a useful interpretation in both the Riemannian and Lorentz settings. Let $f^{*}$ be the quadratic form determined by $g^{*}$. If $g$ and $g^{*}$ are Riemannian metrics, and $S_{p}^{n-1}$ is the $g$-unit tangent sphere to $M$ at a point $p$ in $\mathscr{Q}$, then the restriction of $f^{*}$ to $S_{p}^{n-1}$ assumes its maximum at precisely those $g$-unit tangent vectors that span $\Re$ at $p$.

If $g$ and $g^{*}$ are Lorentz, we can make a similar interpretation of $\Re$. If $H_{p}^{n-1}$ is the set of tangent vectors $X$ in $M_{p}$ such that $g(X, X)=-1$, then at any point $p$ of $\mathscr{D}$, the restriction of $f^{*}$ to $H_{p}^{n-1}$ assumes its maximum at precisely those $g$-unit timelike vectors that span $\Re$ at $p$.

3.2. Properties of the maximal line field. The line field $\mathscr{T}$ is an important key in understanding the structure of $(M, g)$. However, to utilize $\Re$, we first restrict our attention to a special subset $\mathcal{V}$ of $M$. Consider the set $\mathcal{Q}$ of vectors $W$ in the normal bundle $\nu(H)$ that satisfy

(i) $\exp (t W)$ belongs to $\mathscr{Q}$ for all $t$ in the interval $[0,1]$;

(ii) exp is regular at $t W$ for all $t$ in $[0,1]$.

We define $\mathcal{V}$ to be the image of $\mathcal{Q}$ under the exponential map. Thus $\mathcal{V}$ consists of the points on geodesic segments orthogonal to $H$, each segment extending until it either leaves $\mathscr{W}$, or encounters the first focal point of its base point on $H$. One easily sees that $\mathcal{V}$ is an open nonempty subset of $M$.

Our first step in understanding the structure of $(M, g)$ will be to show that the integral curves to $\mathfrak{T}$ in $\mathscr{V}$ are actually geodesics.

LEMma 3.2.1. In $\mathcal{T}$, a unit speed curve $\alpha$ is an integral curve of $\mathfrak{T}$ if and only if $\alpha$ is a geodesic orthogonal to $H$.

Proof. We first assume that $\alpha$ is a unit speed integral curve of $\Re$ with velocity $V(t)$ and that $\alpha(0)$ belongs to $H$.

Since $|g(V(t), V(t))|=1$, the acceleration, $\nabla_{V} V$, of $\alpha$ must be orthogonal to $V$. Moreover, those vectors $g$-orthogonal to $\Re$ are also $g^{*}$-orthgonal to $\Re$. Hence $g^{*}\left(\nabla_{V} V, V\right)=0$. This fact together with the Weyl formula yields

$$
\frac{d}{d t} g^{*}(V(t), V(t))=4\left\{\frac{d}{d t} \ln Q \circ \alpha(t)\right\} g^{*}(V(t), V(t)) .
$$


Thus,

$$
g^{*}(V(t), V(t))=\left[Q^{4} \circ \alpha(t) / Q^{4} \circ \alpha(0)\right] g^{*}(V(0), V(0)) .
$$

Since $\alpha(0)$ lies in $H$, its velocity is given by $V(0)= \pm N= \pm 1 / c N^{*}$. Hence

$$
g^{*}(V(t), V(t))=\left[Q^{4} \circ \alpha(t) / b^{4} c^{2}\right] g(N, N)
$$

where $b=Q(H)$.

On the other hand, consider a unit speed geodesic $\gamma$ in $\mathcal{V}$ that is orthogonal to $H$ and passes through $\alpha(t)$. If we take $Z$ to be the velocity of $\gamma$, then by Proposition 2.1.1,

$$
g^{*}(Z, Z)=\left[Q^{4} \circ \gamma / b^{4} c^{2}\right] g(N, N)
$$

Hence, at $\alpha(t)$ we have $g^{*}(Z, Z)=g^{*}(V, V)$. But from subsection 3.1 we know that $g^{*}(Z, Z)$ and $g^{*}(V, V)$ have the same value if and only if $Z$ also spans $\mathfrak{N}$. Thus, $\gamma$ and $\alpha$ must be tangent at $\alpha(t)$.

Choose an open neighborhood $U$ of $\alpha(t)$ in $\mathcal{V}$ that is the diffeomorphic image of some open subset of $\nu(H)$ under the exponential map. From the above it follows that $\alpha$ must be tangent to the geodesic vector field on $U$ that is the image, under the differential of the exponential map, of the unit vector field tangent to the fibers of $\nu(H)$. Hence $\alpha$ must be a geodesic orthogonal to $H$.

Thus, if $\gamma$ is a maximal unit speed geodesic in $\mathcal{V}$ orthogonal to $H$ with $\gamma(0)$ in $H$, then $\gamma$ must coincide with the maximal unit speed integral curve of $\Re$ that satisfies the same initial conditions. Moreover, since any point in $\mathcal{T}$ lies on a maximal geodesic orthogonal to $H$, it follows that every maximal integral curve of $\Re$ must intersect $H$. Q.E.D.

We next consider the distribution $\mathcal{H}$ on $\mathscr{T}$ of vector fields orthogonal to $\mathscr{N}$. From the Gauss Lemma and Lemma 3.2.1, it follows that if the vector field $t N$ is a local section of the subset $\mathcal{Q}$ of $\nu(H)$ over some open subset of $H$, then the image of this section under the exponential map is a hypersurface of $\mathscr{V}$ which is everywhere orthogonal to $\mathfrak{K}$. Thus $\mathcal{H}$ is integrable. Furthermore, each connected leaf of $\mathcal{H}$ must be at a constant orthogonal distance from $H$ in $\mathcal{V}$.

Every vector field $E$ on $\mathcal{V}$ can be expressed uniquely as $\mathcal{H}(E)+\mathscr{K}(E)$, where $\mathcal{H}(E)$ is the component of $E$ that lies in $\mathcal{H}$, and $\mathscr{T}(E)$ is the component of $E$ in $\Re$. Define the $(0,2)$ tensors $h$ and $m$ on $\mathcal{V}$ by

$$
\begin{aligned}
h(X, Y) & =g(\mathcal{H}(X), \mathscr{H}(Y)), \\
m(X, Y) & =g(\mathfrak{K}(X), \mathfrak{K}(Y))
\end{aligned}
$$

for vector fields $X$ and $Y$ on $\mathcal{V}$. We can thus express $g$ on $\mathcal{T}$ as $h+m$.

Proposition 3.2.2. If $L$ is a leaf of the distribution $\mathcal{H}$, then $Q$ is constant along $L$. Furthermore, throughout $\mathcal{W}$ the metric $g^{*}$ is given by

$$
g^{*}=\left[Q^{2} / b^{2}\right] h+\left[Q^{4} / b^{4} c^{2}\right] m
$$

where $b$ denotes $Q(H)$.

Proof. We first show that if $X$ is in $\mathcal{H}$ then $X(Q)=0$. 
Let $\gamma$ be a geodesic orthogonal to $H$ with velocity $V$. Choose a vector field $X$ parallel along, and orthogonal to, $\gamma$. Since $\gamma$ is an integral curve of $\Re$, we have $g^{*}(V, X)=0$. But, by Proposition 2.1.1,

$$
\begin{aligned}
g^{*}(V(t), X(t))= & \frac{Q^{3} \circ \gamma(t)}{b^{3}}\left[\int_{0}^{t}(X \ln Q) \circ \gamma(s) d s\right] g^{*}(V(0), V(0)) \\
& +\frac{Q^{3} \circ \gamma(t)}{b^{3}} g^{*}(V(0), X(0)) .
\end{aligned}
$$

Thus for every choice of $t$,

$$
\int_{0}^{t}(x \ln Q) \circ \gamma(s) d s=0 .
$$

Therefore $X(Q)=0$ and hence $Q$ must be constant along each leaf of $\mathcal{H}$.

If $X_{q}$ and $Y_{q}$ are any vectors orthogonal to $\mathfrak{N}$ at a point $q$ in $\mathcal{V}$, they may be viewed as the parallel translation of some vectors $X_{H}$ and $Y_{H}$ tangent to $H$ along some geodesic orthogonal to $H$. Once again employing Proposition 2.1.1, we have

$$
g^{*}\left(X_{q}, Y_{q}\right)=\left[Q^{2}(q) / b^{2}\right] g^{*}\left(X_{H}, Y_{H}\right) .
$$

But $g^{*}\left(X_{H}, Y_{H}\right)=g\left(X_{H}, Y_{H}\right)=g\left(X_{q}, Y_{q}\right)$. Hence

$$
g^{*}\left(X_{q}, Y_{q}\right)=\left[Q^{2}(q) / b^{2}\right] g\left(X_{q}, Y_{q}\right) \text {. }
$$

By Proposition 2.1.1 and the fact that $1 / c N^{*}=N$, it follows that if $V$ and $W$ are in M, then

$$
g^{*}(V, W)=Q^{4} / b^{4} c^{2} g(V, W) .
$$

Since $\mathcal{H}$ and $\Re$ are also $g^{*}$-orthogonal, we have

$$
g^{*}(E, F)=g^{*}(\mathcal{H}(E), \mathcal{H}(F))+g^{*}(\mathscr{T}(E), \mathfrak{T}(F))
$$

for all vector fields $E$ and $F$ on $\mathcal{V}$. Combining (2), (3) and (4) yields equation (1). Q.E.D.

The second fundamental form of the leaves of $\mathcal{H}$ can be used to give a $(1,2)$ tensor, $T$, on $\mathcal{T}$. As in $\S 1$, we define $T$ by

$$
T(E, F)=\mathscr{N}\left[\nabla_{\mathcal{K}(E)} \mathcal{H}(F)\right]+\mathcal{H}\left[\nabla_{\mathcal{K}(E)} \mathfrak{N}(F)\right]
$$

for any vector fields $E$ and $F$ on $\mathcal{T}$. One easily verifies that $T$ is multilinear over the $C^{\infty}$-functions on $\mathcal{T}$, and is indeed a tensor on $\mathcal{T}$. The following proposition summarizes the properties of $T$.

Proposition 3.2.3. Suppose $X$ and $Y$ are vector fields belonging to $\mathcal{H}$ and $Z$ is a vector field in $\mathfrak{T}$. Then

$$
T(X, Y)=\left[\Re(\ln Q) b^{2} c^{2} /\left(b^{2} c^{2}-Q^{2}\right)\right][g(X, Y) / g(\Re, \Re)] \Re
$$

where $\mathfrak{N}$ is a unit vector field in $\mathfrak{\Re}$.

(ii) $T(X, Z)=\left[g\left(\nabla_{X} Z, X\right) / g(X, X)\right] X=\left[b^{2} c^{2} /\left(Q^{2}-b^{2} c^{2}\right)\right] Z(\ln Q) X$. 
ReMARK. The denominator of (i) above cannot be zero in $\Upsilon$; for if $Q^{2} b^{2}-c^{2}=0$, it then follows from Proposition 3.2.2 that $g^{*}=\left(Q^{2} / b^{2}\right) g$. However, this contradicts the requirement that $G$ has a simple maximum eigenvalue throughout $\mathcal{T}$.

Proof of Proposition 3.2.3. (i) Let $\mathfrak{T}$ be a unit vector field in $\mathscr{N}$. By the definition of $T$ and the hypothesis that $X$ and $Y$ belong to $\mathcal{H}$, we have

$$
T(X, Y)=\left[g\left(\nabla_{X} Y, \mathfrak{R}\right) / g(\mathfrak{x}, \mathfrak{x})\right] \Re .
$$

Since $X$ and $Y$ belong to $\mathcal{H}$ we know that $g^{*}(X, \mathfrak{N})=g^{*}(Y, \mathcal{X})=0$. Differentiating $g^{*}(Y, \mathcal{X})$ by $X$ and applying the Weyl formula gives

$$
0=g^{*}\left(\nabla_{X} Y, \mathfrak{X}\right)+g^{*}\left(Y, \nabla_{X} \mathfrak{X}\right)+\mathfrak{X}(\ln Q) g^{*}(X, Y) .
$$

Routine algebra, Proposition 3.2.2 and the observation that $g\left(\nabla_{X} Y, \mathcal{X}\right)=$ $-g\left(Y, \nabla_{X} \Re\right)$ when applied to equations (1) and (2) yield the assertion of (i).

(ii) Since $T$ is a tensor on $\mathcal{V}$ we may choose any convenient extension of $Z_{p}$ when computing $T(X, Y)_{p}$. Thus we assume that $Z$ has constant length. Consequently, $X g(Z, Z)=0$ and $\nabla_{X} Z$ must be orthogonal to $\Re$.

Choose $X, \ldots, X_{n-1}$ locally to be a orthogonal collection of vector fields spanning $\mathcal{H}$. Then $g\left(\nabla_{X} Z, X_{i}\right)=-g\left(\nabla_{X} X_{i}, Z\right)$. Applying (2) from above, together with Proposition 3.2.2, we have

$$
g\left(\nabla_{X} Z, X_{i}\right)=-\left[b^{2} c^{2} /\left(b^{2} c^{2}-Q^{2}\right)\right] Z(\ln Q) g\left(X, X_{i}\right),
$$

from which (ii) follows. Q.E.D.

The leaves of the distribution $\mathcal{H}$ and the geodesics tangent to the maximal line field $\mathscr{R}$ provide the framework necessary for establishing the warped product structure of $(\mathfrak{V}, g)$. The integral submanifolds of $\mathcal{H}$ will be the leaves of the warped product; the normal geodesics will be the fibers of the product. It is clear that, locally, $\mathcal{H}$ and $\mathfrak{N}$ provide a manifold product structure. To prove that they, in fact, provide a local warped product structure, we need to show that translation along normal geodesics carries a leaf of $\mathcal{H}$ homothetically to a leaf of $\mathcal{H}$.

Proposition 3.2.4. If $p$ is a point of $\mathcal{T}$, then there exists a neighborhood Of of $p$, an open subset $U$ of $H$, an interval $I$, and a function $f$ on I such that $(\mathcal{Q}, g)$ is isometric to $\left(U, h_{0}\right) \times_{f}(I, m)$, where $h_{0}$ is the metric induced on $U$ by $g$, and $m$ is the standard metric on $I$.

Proof. Recall the definition of the open subset $\mathscr{U}$ of $\nu(H)$ used to define $\mathcal{V}$ at the beginning of this subsection. Let $U$ be a connected open subset of $H$ and let $I$ be an interval containing zero such that (i) $U \times I$ is a local trivialization of $\nu(H)$ under the correspondence that identifies $(x, t)$ with $t N_{x}$, and (ii) the trivialization $U \times I$ is contained in $\mathscr{U}$ and is carried diffeomorphically to a neighborhood of $p$ by the exponential map.

Define $f$ on $I$ by

$$
f(t)=\left[\frac{Q^{2}(t)-b^{2} c^{2}}{\left(1-c^{2}\right) Q^{2}(t)}\right]^{1 / 2}
$$


where $Q(t)=Q(\exp [U \times\{t\}])$. The exponential map will provide the desired isometry between $\left(U, h_{0}\right) \times_{f}(I, m)$ and $\mathscr{Q}$.

To establish that this map is indeed an isometry, it suffices to show that exp carries the leaves of $\left(U, h_{0}\right) \times_{f}(I, m)$ isometrically to the leaves of $\mathcal{H}$ in $\mathscr{W}$. Consider a vector field $J_{0}$ on $U$ and let $\tilde{J}$ be the canonical vector field it determines on $U \times I$. Similarly let $\tilde{Z}$ denote the canonical extension of the unit vector field $\partial / \partial t$ on $I$ to $U \times I$; note that $[\tilde{J}, \tilde{Z}]=0$. If we let $\hat{g}$ denote the warped product metric of $\left(U, h_{0}\right) \times{ }_{f}(I, m)$, then on the leaf $U \times\{t\}$,

$$
\hat{g}(\tilde{J}, \tilde{J})=\left\{\left[Q^{2}(t)-b^{2} c^{2}\right] /\left[\left(1-c^{2}\right) Q^{2}(t)\right]\right\} h_{0}\left(J_{0}, J_{0}\right) .
$$

Let $J$ and $Z$ denote the images of $\tilde{J}$ and $\tilde{Z}$ under the differential of the exponential map. The vector field $J$ is a Jacobi field along the geodesics normal to $H$ in $\mathscr{U}$; the vector field $Z$ is the velocity field of geodesics. Since $[\tilde{J}, \tilde{Z}]=0$, it follows that $[J, Z]=0$ and hence $\nabla_{Z} J=\nabla_{J} Z$. Consequently, by Proposition 3.2.3 and the fact that $Z$ has constant length on $\mathcal{W}$, it follows that

$$
\nabla_{Z} J=T(J, Z)=\left[b^{2} c^{2} /\left(Q^{2}-b^{2} c^{2}\right)\right] Z(\ln Q) J .
$$

We may assume that $U$ is sufficiently small so as to allow the existence of an orthonormal basis field $\hat{v}_{1}, \ldots, \hat{v}_{n-1}$ on $U$. Let $V_{1}, \ldots, V_{n-1}$ be their extensions to थf via parallel translation along normal geodesics. Then there are functions $J_{1}, \ldots, J_{n-1}$ on थै such that $J=\sum_{i=1}^{n-1} J_{i} V_{i}$. Identifying $U$ with $U \times I$ by the exponential map, we may regard the $J_{i}$ as functions on $U \times I$.

Since $V_{i}$ is parallel with respect to $Z$ it follows that

$$
\nabla_{Z} J=\sum_{i=1}^{n-1} Z\left(J_{i}\right) V_{i}
$$

Recall that $Q^{2}-b^{2} c^{2}$ is positive on $\mathcal{T}$. Combining this fact with the definition of $Z$, equations (1) and (2) yield

$$
\frac{\partial J_{i}}{\partial t}=\left[b^{2} c^{2} /\left(Q^{2}-b^{2} c^{2}\right)\right] \frac{\partial(\ln Q)}{\partial t} J_{i}
$$

But one can easily verify that

$$
\left[b^{2} c^{2} /\left(Q^{2}-b^{2} c^{2}\right)\right] \frac{\partial}{\partial t} \ln Q=\frac{1}{2} \frac{\partial}{\partial t} \ln \left[\frac{Q^{2}-b^{2} c^{2}}{Q^{2}}\right]
$$

Therefore

$$
J_{i}(x, t)=\left[\frac{Q^{2}(t)-b^{2} c^{2}}{\left(1-c^{2}\right) Q^{2}(t)}\right]^{1 / 2} J_{i}(x, 0) .
$$

Thus, if $J_{*}$ denotes the parallel translation of $J_{0}$ along geodesics orthogonal to $H$, we have

$$
J=\left[\frac{Q^{2}-b^{2} c^{2}}{\left(1-c^{2}\right) Q^{2}}\right]^{1 / 2} J_{*}
$$


Hence the exponential map carries the fibers of $\left(U, h_{0}\right) \times_{f}(I, m)$ isometrically to the leaves of $\mathcal{H}$ in $\mathcal{W}$. Q.E.D.

Remark. By applying Propositions 3.2.2 and 1.3.3, we see that (थ्W, $\left.g^{*}\right)$ is also a warped product manifold.

3.3. Visibility and its implications. In the last subsection, we limited our attention to the set $\mathfrak{V}$, which is foliated by the geodesics orthogonal to $H$. We next investigate the complement of $\mathfrak{V}$ in $M$. For this purpose we shall require assumptions concerning the global relationship between $M$ and $H$.

Definition 3.3.1. We say that $(M, g)$ is weakly visible from $H$ if the exponential map is surjective from $\nu(H)$ to $M$. We say that $(M, g)$ is visible from $H$ if for each point $p$ of $M$ there is a vector $V$ in $\nu(H)$ such that (i) $\exp (V)=p$ and (ii) the exponential map, when restricted to $\nu(H)$, is regular at $t V$ for each $t$ in the interval $[0,1)$.

Note that weak visibility does not imply visibility. For example, let $S^{n}$ be the unit sphere in $E^{n+1}$, and let $S^{n-1}$ be a great sphere of $S^{n}$. If $p$ is any point in $S^{n}-S^{n-1}$ that is not equidistant from the points of $S^{n-1}$, then $S^{n}-\{p\}$ is weakly visible from $S^{n-1}$ but is not visible from $S^{n-1}$.

The condition that $M$ be visible from $H$ is a natural restriction upon $M$ in either the Riemannian or Lorentz setting. If $(M, g)$ is a complete Riemannian manifold and $H$ is a complete hypersurface, then $(M, g)$ is visible from $H$. In the Lorentz setting, if $H$ is a Cauchy hypersurface in $(M, g)$ then $M$ is visible from $H$.

In addition to the idea of visibility, the concept of a conformal point will play an important role in the determination of the structure of $(M, g)$.

Definition 3.3.2. A point $p$ of $M$ is said to be a conformal point of $M$ if the metrics $g$ and $g^{*}$ are conformally related at $p$.

Throughout this subsection, we focus our attention on the situation in which $M$ has no conformal points. We shall see in the final subsection of this work that under the assumption of visibility, the number of conformal points is severly restricted. Moreover, if $M$ does indeed possess conformal points, $H$ is completely determined.

Proposition 3.3.3. If $(M, g)$ is weakly visible from $H$, and $M$ contains no conformal points, then $g$ and $g^{*}$ are locally warped product metrics on $M$.

Proof. By Proposition 3.2.4, it suffices to show that $\mathfrak{T}$ coincides with $M$.

Suppose there is a point $p$ of $M$ that is not contained in $\widetilde{T}$. Let $\gamma$ be a geodesic orthogonal to $H$ that contains $p$. Since $p$ is not in $\mathscr{V}$, there must be a first point at which $\gamma$ leaves $\mathscr{V}$; call it $q$. By the definition of $\mathfrak{V}$, the point $q$ must be either the first focal point of $H$ along $\gamma$ or a conformal point. The latter case is ruled out by hypothesis, hence there is a nonzero Jacobi field, $J$, along $\gamma$ that vanishes at $q$. From equation (3) of the preceding proof, such a Jacobi field, when restricted to the portion of $\gamma$ in $\mathscr{V}$, can be expressed as

$$
J(t)=\left\{\sqrt{\left(Q^{2} \circ \gamma(t)-b^{2} c^{2}\right) / Q^{2} \circ \gamma(t)}\right\} X(t)
$$

where $X(t)$ is the parallel translation of $J(0)$ along $\gamma$. From this expression it is clear that $H$ vanishes at $q$ only if $Q^{2}(q)=b^{2} c^{2}$. Hence, by Proposition 3.2.2, the point $q$ 
must be a conformal point of $M$, a contradiction. Therefore, $\mathcal{T}$ and $M$ must coincide. Q.E.D.

REMARK. The above proof shows that if a geodesic normal to $H$ escapes from $\mathcal{T}$, the point at which it leaves $\mathscr{V}$ is both a focal point of $H$ and a conformal point of $M$. Furthermore, it follows that if $M$ is visible from $H$, the only points of $M$ not contained in $\mathcal{V}$ are conformal points.

We next give conditions which insure that $M$ is globally a warped product.

Proposition 3.3.4. Suppose that $M$ is weakly visible from $H$ and has no conformal points. If each geodesic normal to $H$ intersects $H$ in only one point, and each normal vector field of constant length intersects the domain of the exponential map in a connected set, then $(M, g)$ and $\left(M, g^{*}\right)$ can be isometrically imbedded as open subsets of warped product manifolds.

Proof. As in the preceding proposition, we have $M=\mathfrak{T}$. There are two cases to be considered: Case (1) in which no geodesic orthogonal to $H$ is periodic, and Case (2) in which there exists a periodic geodesic orthogonal to $H$.

Case (1). If there are no periodic geodesics normal to $H$, then for each $p$ in $M$ there is a unique point $(x(p), t(p))$ in $H \times R$ such that $\exp \left[t(p) N_{x(p)}\right]=p$. Define constants $\alpha$ and $\beta$ by

$$
\alpha=\inf _{p \in M} t(p), \quad \beta=\sup _{p \in M} t(p) .
$$

Let $I$ denote the interval $(\alpha, \beta)$. Consider any $t$ in $I$; by assumption the vector field $t N$ intersects the domain of definition of the exponential map in a nonempty connected set, say $U_{t}$. Since $\exp \left(U_{t}\right)$ is a leaf of $\mathcal{H}$, on which $Q$ is constant, we may define a function $f$ on $I$ by

$$
f(t)=\left[\frac{Q^{2} \circ \exp \left(U_{t}\right)-b^{2} c^{2}}{\left(1-c^{2}\right) Q^{2} \circ \exp \left(U_{t}\right)}\right]^{1 / 2} .
$$

The map $\mathcal{E}$ from $(M, g)$ to $(H, h) \times_{f}(I, m)$ given by $\mathcal{E}(p)=(x(p), t(p))$ is injective. Furthermore, from the proof of Proposition 3.2.4, it is clear that $\mathcal{E}$ is a local isometry. Therefore it must be an isometric imbedding. By applying Propositions 1.3.3 and 3.2.2, we can find an isometric imbedding of $\left(M, g^{*}\right)$ into a warped product of $H$ and an interval.

Case (2). If there is a closed geodesic normal to $H$, we simply replace the interval $I$ by a circle having the same length as the closed geodesic and proceed as above. Q.E.D.

Although the hypothesis of Proposition 3.3.4 concerning the connectivity of the image of vector fields in the domain of the exponential map is somewhat awkward, it is satisfied if $(M, g)$ is a complete, Riemannian manifold and $H$ is a complete hypersurface. Letting $g_{H}$ stand for the metric induced on $H$ by $g$, we have the following

THEOREM 3.3.5. Suppose that $g$ and $g^{*}$ are projectively equivalent Riemannian metrics on the manifold $M$, aligned along a hypersurface, $H$, admitting a global choice of unit normal field. Suppose further that $(M, g)$ and $\left(H, g_{H}\right)$ are complete. If each 
geodesic orthogonal to $H$ intersects $H$ at only one point and $M$ contains no conformal points, then $(M, g)$ is isometric to a Riemannian warped product of $\left(H, g_{h}\right)$ and $a$ complete one-dimensional Riemannian manifold.

REMARK. One can easily construct examples that demonstrate the necessity in Theorem 3.3.5 of the hypothesis that each geodesic orthogonal to $H$ intersect $H$ at only one point. Suppose that $(H, h)$ is a complete Riemannian manifold that admits a nontrivial self-isometry $\psi$, and that $f$ is a smooth positive function on $R$ (the reals) with period $P$. Let $m$ be the standard metric on $[0, P]$. Construct $M$ from $H \times[0, P]$ by identifying $(x, 0)$ with $(\psi(x), P)$. By Proposition 1.3.3, the metrics $g=f^{2}(t) h+m$ and

$$
g^{*}=\frac{2 f^{2}(0) f^{2}(t)}{f^{2}(0)+f^{2}(t)} h+\frac{2 f^{4}(0)}{\left(f^{2}(0)+f^{2}(t)\right)^{2}} m
$$

are projectively equivalent on $M$. Furthermore, they are aligned along $H \times\{0\}$ and are both complete on $M$. Finally, $H \times\{0\}$ admits a unit normal field and $M$ has no conformal points.

We end this subsection with a theorem concerning strictly convex, complete Riemannian manifolds.

DEFInITION 3.3.6. A Riemannian manifold $(M, g)$ is said to be strictly convex if for each pair of points in $M$ there is a unique geodesic that joins them and each geodesic segment is distance realizing between its endpoints.

Theorem 3.3.7. Suppose that $(M, g)$ is a strictly convex, complete Riemannian manifold, and that $g$ and $g^{*}$ are projectively equivalent metrics aligned along $a$ complete hypersurface $H$ that admits a global unit normal field. If $M$ has no conformal points, then $(M, g)$ is isometric to a warped product manifold of $H$ with $E^{1}$.

Proof. There are no closed geodesics in $M$; thus by Theorem 3.3.5 it suffices to show that each geodesic orthogonal to $H$ intersects $H$ at only one point.

Suppose that some unit speed geodesic $\gamma$ orthogonal to $H$ intersects $H$ at two consecutive points, $\gamma(0)$ and $\gamma(r)$. Since $Q \circ \gamma(0)=Q \circ \gamma(r)$, there is a value $t$ between 0 and $r$ such that $(d / d t) Q \circ \gamma(t)=0$. Thus by Proposition 3.2.3, the maximal integral submanifold $H_{t}$ of $\mathcal{H}$ passing through $\gamma(t)$ must be a totally geodesic hypersurface of $(M, g)$. This hypersurface is the homothetic image of $H$ under the map which carries $x$ to $\exp \left[t N_{x}\right]$, where $N$ is the extension of the initial velocity of $\gamma$ to a unit normal field on $H$. Thus $H_{t}$ is a complete hypersurface in $(M, g)$. But a complete totally geodesic hypersurface of a strictly convex Riemannian manifold separates the manifold into two disjoint open convex sets. Since $\gamma(0)$ and $\gamma(r)$ lie on opposite sides of $H_{t}$, and $H$ cannot intersect $H_{t}$, the hypersurface $H$ cannot be connected, a contradiction.

Therefore, every geodesic orthogonal to $H$ intersects $H$ at precisely one point. Q.E.D.

3.4. A Clairaut theorem. As Bishop has shown in his paper [2], the geodesics of a warped product manifold satisfy a "Clairaut Theorem". Thus the first result of this 
section could be deduced from Bishop's work. [While Bishop's results were obtained for Riemannian metrics, they can easily be generalized to the semi-Riemannian setting.] In the context of this work, we can easily provide an independent proof, and do so for the sake of completeness.

THEOREM 3.4.1. Suppose that $g$ and $g^{*}$ are projectively equivalent metrics on $M$ and that they are aligned along the hypersurface $H$. Let $\mathcal{T}$ be the open neighborhood of $H$ defined in subsection 3.2. If $\gamma$ is a geodesic in $\mathcal{V}$ with velocity $V(t)$, and $\mathcal{H}[V(t)]$ is the component of $V(t)$ tangent to $\mathcal{H}$, then the function

$$
\frac{b^{2} c^{2}-Q^{2} \circ \gamma(t)}{Q^{2} \circ \gamma(t)} g(\mathcal{H}[V(t)], \mathcal{H}[V(t)])
$$

is constant.

Proof. In order to keep notation manageable let $Q(t)$ stand for $Q \circ \gamma(t)$, $E(t)=\mathscr{H}[V(t)]$, and $Y(t)$ be the component of $V(t)$ tangent to the line field $\mathscr{T}$.

By Proposition 2.1.1,

$$
g^{*}(V(t), V(t))=\left[Q^{4}(t) / Q^{4}(0)\right] g^{*}(V(0), V(0)) .
$$

But by Proposition 3.2.2 we know that for a geodesic $\gamma$ in $\mathcal{V}$,

$$
g^{*}(V(t), V(t))=\frac{Q^{2}(t)}{b^{2}} g(E(t), E(t))+\frac{Q^{4}(t)}{b^{4} c^{2}} g(Y(t), Y(t)) .
$$

Thus, from (1) and (2) we have

$$
\begin{aligned}
& \frac{Q^{4}(t)}{Q^{4}(0)}\left\{\frac{Q^{2}(0)}{b^{2}} g(E(0), E(0))+\frac{Q^{4}(0)}{b^{4} c^{2}} g(Y(0), Y(0))\right\} \\
& =\frac{Q^{2}(t)}{b^{2}} g(E(t), E(t))+\frac{Q^{4}(t)}{b^{4} c^{2}} g(Y(t), Y(t)) .
\end{aligned}
$$

Simplifying equation (3) and rearranging terms we obtain

$$
\begin{aligned}
\frac{Q^{4}(t)}{b^{2} Q^{2}(0)}\left\{\frac{b^{2} c^{2}-Q^{2}(0)}{b^{2} c^{2}} g(\right. & E(0), E(0)) \\
& \left.+\frac{Q^{2}(0)}{b^{2} c^{2}}[g(E(0), E(0))+g(Y(0), Y(0))]\right\} \\
= & \frac{Q^{2}(t)}{b^{2}}\left\{\frac{b^{2} c^{2}-Q^{2}(t)}{b^{2} c^{2}} g(E(t), E(t))\right. \\
& \left.+\frac{Q^{2}(t)}{b^{2} c^{2}}[g(E(t), E(t))+g(Y(t), Y(t))]\right\} .
\end{aligned}
$$

But since $V$ is the velocity of a geodesic,

$$
g(E(t), E(t))+g(Y(t), Y(t))=g(V(t), V(t))
$$


is constant. Thus simplifying (4) we have

$$
\frac{b^{2} c^{2}-Q^{2}(t)}{Q^{2}(t)} g(E(t), E(t))=\frac{b^{2} c^{2}-Q^{2}(0)}{Q^{2}(0)} g(E(0), E(0)) .
$$

Since the right-hand side of this equation is constant, we have the desired result. Q.E.D.

Theorem 3.4.1 has useful geometric interpretations in both the Lorentz and Riemannian settings.

Recall Clairaut's Theorem for surfaces of revolution: if $\gamma$ is a geodesic on a surface of revolution, $\theta(t)$ is the angle between $\gamma$ and the meridian passing through $\gamma(t)$, and $r(t)$ is the distance from $\gamma$ to the axis of revolution of the surface, then $r(t) \sin \theta(t)$ is constant.

In the Riemannian setting of Theorem 3.4.1 the value $g(\mathcal{H}[V(t)], \mathscr{H}[V(t)])$ can be regarded as $|V(t)| \sin \theta(t)$, where $\theta(t)$ is the angle between $V(t)$ and $\mathfrak{R}$. Since $|V(t)|$ is constant, it follows that $\sqrt{Q^{2}(t)-b^{2} c^{2} / Q^{2}(t)} \sin \theta(t)$ is constant along $\gamma$ in $\mathfrak{T}$. Thus in the Riemannian case the theorem can be viewed as a generalization of Clairaut's Theorem to higher dimensional spaces.

In the Lorentz case $g(\mathcal{H}[V(t)], \mathcal{H}[V(t)])$ does not measure the sine of an angle between $V(t)$ and $\mathscr{N}$, but measures the square of the momentum of $\gamma$ as observed by the family of freely falling observers that $\Re$ determines. If $\hat{p}(t)$ denotes this observed momentum, Theorem 3.4.1 implies that $\sqrt{Q^{2}(t)-b^{2} c^{2} / Q^{2}(t)}|\hat{p}(t)|$ is constant.

Just as Clairaut's Theorem enables one to deduce global properties of the geodesic of a surface of revolution, Theorem 3.4.1 enables us to establish the following useful corollary.

Corollary 3.4.2. Suppose that $(M, g)$ is visible from $H$. Then a geodesic $\gamma$ in $\mathcal{T}$ can escape from $\mathfrak{T}$ only if it is orthogonal to $H$.

Proof. Suppose $\gamma$ lies in $\mathcal{T}$ and escapes from $\mathcal{T}$. From Theorem 3.4.1 we know that if $V(t)$ is the velocity of $\gamma$, then

$$
\left[\left(Q^{2} \circ \gamma(t)-b^{2} c^{2}\right) / Q^{2} \circ \gamma(t)\right] g(\mathcal{H}[V(t)], \mathcal{H}[V(t)])
$$

is constant at those points of $\gamma$ in $\mathcal{V}$. Let $q$ be the first point at which $\gamma$ leaves $\mathcal{V}$. Since $M$ is visible from $H$, there is a geodesic $\alpha$ orthogonal to $H$ containing $q$ such that there are no focal points of $H$ on $\alpha$ between $H$ and $q$. Consequently by the Remark following Proposition 3.3.3, $q$ must be a focal point of $H$ and a conformal point. Moreover, since it is the first such point along $\alpha$, we must have $Q^{2}(q)-b^{2} c^{2}$ $=0$. But since (1) is constant, it must be identically zero. Therefore, $\gamma$ must be tangent to $\mathfrak{T}$ and orthogonal to $H$. Q.E.D.

3.5. The implications of conformal points. In subsection 3.3, we saw that if $M$ has no conformal points and is visible (or weakly visible) from $H$, then $(M, g)$ is locally a warped product manifold. In this subsection we shall see that visibility strictly limits the number of conformal points in $M$ and that if $M$ does have conformal points, the structure of $H$ is uniquely determined. In addition, we shall see that, unlike the 
preceding sections, the results for Lorentz manifolds are not merely analogues of the Riemannian results.

THEOREM 3.5.1. Suppose that $g$ and $g^{*}$ are projectively equivalent Lorentz metrics on a manifold, $M$, and that they are aligned along some Riemannian hypersurface $H$. If $(M, g)$ is visible from $H$, then $M$ contains no conformal points.

Proof. Suppose $p$ is a conformal point of $M$. Let $B$ be a strictly convex neighborhood of $p$ and let $q$ be any point of this neighborhood. Since $M$ is visible from $H$, there is a geodesic $\gamma$ joining $q$ to $H$, that is orthogonal to $H$ and whose interior points lie in $\widetilde{T}$. Let $q^{*}$ be an interior point of $\gamma$ that lies in $B$. Because $q^{*}$ lies in $B$, there is a geodesic (in $B$ ) joining $q^{*}$ to $p$. Since $q^{*}$ also lies in $\mathcal{T}$, it follows from Corollary 3.4.2 that this geodesic is orthogonal to $H$ and hence coincides with $\gamma$. Thus the geodesic from $p$ to $q$ must be $\gamma$, which is timelike. Therefore, every vector tangent to $M$ at $p$ is timelike, contradicting the hypothesis that $g$ is Lorentz. Thus there can be no conformal points in $M$. Q.E.D.

We now turn our attention to the Riemannian setting. From the constant curvature examples of $\S 1$, it is clear that in this case the metrics $g$ and $g^{*}$ may indeed be conformally related at some point of $M$. However, in each of these examples the hypersurface $H$ is a standard sphere. If conformal points exist, this condition upon $H$ is necessary.

THEOREM 3.5.2. Let $g$ and $g^{*}$ be projectively equivalent Riemannian metrics on $M$, aligned along some hypersurface $H$ that admits a global unit normal field. If $M$ is visible from $H$ and contains a conformal point, then $H$ is isometric to a standard sphere.

Proof. First note that it follows as in the proof of Theorem 3.5.1 that every geodesic ray from a conformal point $p$ intersects $H$ orthogonally. Moreover, the interior of a distance realizing geodesic segment joining $p$ to $H$ contains no conformal points, and hence lies in $\mathcal{V}$. Since $\mathcal{H}$ is orthogonal to the geodesics orthogonal to $H$, it follows from the Gauss Lemma that the leaves of $\mathcal{H}$ between $p$ and $H$ are metric spheres centered about $p$.

Let $\varepsilon_{r}$ be the map from $M_{p}$ to $M$ given by $\varepsilon_{r}(V)=\exp [r V]$, for all $V$ in $M_{p}$ such that $r V$ is in the domain of the exponential map. If $R$ is the distance of $H$ from $p$, then $\mathcal{E}_{r}$ maps $S_{p}^{n-1}$ [the unit tangent sphere to $M$ at $p$ ] onto $H$.

Because $H$ has a global unit normal field, $\mathcal{E}_{r}$ is globally injective from $S_{p}^{n-1}$ to $H$. To show this we suppose there are two vectors in $S_{p}^{n-1}$, say $V$ and $W$, such that $\mathcal{E}_{R}(V)=\mathcal{E}_{R}(W)$. Consider the two geodesics, $\gamma_{V}(t)=\mathcal{E}_{R}(t V)$ and $\gamma_{W}(t)=\mathcal{E}_{R}(t W)$. Since there is only one geodesic through $\mathcal{E}_{R}(V)$ orthogonal to $H$, the velocities of $\gamma_{V}$ and $\gamma_{W}$ must be the same or opposite at $\mathcal{E}_{R}(V)$. Let $Z$ be the outward pointing unit normal field on $S_{p}^{n-1}$ in $M_{p}$. Then $d \mathcal{E}_{R}\left(Z_{V}\right)$ and $d \mathcal{E}_{R}\left(Z_{W}\right)$ are the velocities of $\gamma_{V}$ and $\gamma_{W}$ at $\mathcal{E}_{R}(V)$. Certainly, $d \mathcal{E}_{R}(Z)$ is a vector field along the map $\mathcal{E}_{R}$ which is everywhere orthogonal to $H$. Furthermore, if $N$ is a unit normal field on $H$, then $g\left(d \mathcal{E}_{R}(Z), N\right)$ is continuous and hence constant on $S_{p}^{n-1}$. Therefore, $d \mathcal{E}_{R}\left(Z_{V}\right)=$ $d \mathcal{E}_{R}\left(Z_{W}\right)$. Consequently, $V$ and $W$ must be equal. Similarly, it follows that, if $r$ is between zero and $R$, then $\mathcal{E}_{r}$ maps $S_{p}^{n-1}$ bijectively to the metric sphere, $S(p ; r)$, of radius $r$ about $p$. 
Let $g^{r}$ denote the metric induced by $g$ on $S(p ; r)$. One can easily verify that, for any Riemannian manifold $(M, g)$, the standard metric on $S_{p}^{n-1}$ is equal to the limit metric given by $\lim _{r \rightarrow 0}\left(1 / r^{2}\right) \mathcal{E}_{r}^{*} g^{r}$, where $\mathcal{E}_{r}^{*} g^{r}$ denotes the pullback of along $\mathcal{E}_{r}$ of the metric $g^{r}$. However, in our warped product setting, the spheres $\left(S(p ; r), g^{r}\right)$ with radius less than or equal to $R$, are homothetically equivalent to one another. Thus each such sphere must be homothetically equivalent to the standard $(n-1)$-sphere. Q.E.D.

The proof of Theorem 3.5.2 also yields the following

COROllary 3.5.3. If $(M, g)$ is visible from $H$, then the set of conformal points consists of at most two points.

Proof. We saw in the proof of Theorem 3.5.2 that if $p$ is a conformal point, then there is a choice of normal field $N$ along $H$ such that every geodesic from $H$ tangent to $N$ passes through $p$, always at the same distance from $H$. Furthermore, no conformal point occurs between $H$ and $p$. Since there are only two choices of normal field to $H$, there can be at most two conformal points. Q.E.D.

The following theorems summarize the implications of the existence of conformal points.

THEOREM 3.5.4. Let $g$ and $g^{*}$ be projectively equivalent Riemannian metrics on a manifold, $M$, aligned along some hypersurface $H$ which admits a global unit normal field. Suppose that $(M, g)$ is complete and visible from $H$. If $M$ contains exactly one conformal point $p$ and each geodesic orthogonal to $H$ in $M-\{p\}$ intersects $H$ in precisely one point, then $M$ is diffeomorphic to $R^{n}$. Furthermore, $M-\{p\}$ is isometric to the warped product of the standard $(n-1)$-sphere with the positive real numbers.

Proof. Let $p$ be the conformal point of $M$ and let $q$ be any other point of $M$. Since there is a unique geodesic in $\mathcal{V}$ orthogonal to $H$ containing $q$, it follows that there is a unique vector $V(q)$ in $M_{p}$ such that $\exp [V(q)]=q$. Furthermore, since $p$ is the only conformal point of $M$, it can have no conjugate points. Hence, the exponential map is regular at $V(q)$. Therefore, the map from $M$ to $M_{p}$ that carries $q$ to $V(q)$ is bijective and regular. Identifying $M_{p}$ with $R^{n}$, this map gives the desired diffeomorphism. The warped product structure of $(M-\{p\}, g)$ follows from Theorem 3.5.2 and Proposition 3.3.4. Q.E.D.

THEOREM 3.5.5. Let $g$ and $g^{*}$ be projectively equivalent Riemannian metrics on $M$, aligned along some hypersurface $H$ which admits a global unit normal field. If $(M, g)$ is visible from $H$ and contains two conformal points, $p$ and $q$, then $M$ is diffeomorphic to a sphere. Moreover, $M-\{p, q\}$ is isometric to a warped product of the standard $(n-1)$-sphere with an interval.

Proof. Let $D$ be the distance between $p$ and $q$. Let $p_{*}$ be any point of the sphere $S^{n}\left(\pi^{2} / D^{2}\right)$ and let $\theta$ be an orthogonal transformation from $M_{p}$ to $S^{n}\left(\pi^{2} / D^{2}\right)_{p_{*}}$. Finally, let $q_{*}$ be the point antipodal to $p_{*}$. For each $x$ in $M-\{q\}$, there is a unique $V(x)$ in $M_{p}$ such that $\exp [V(x)]=x$ and $|V(x)|<D$. Define a function $f$ mapping $M$ onto $S^{n}\left(\pi^{2} / D^{2}\right)$ by

$$
f(x)=\exp ^{*}[\mathcal{O}(V(x))] \text { for } x \neq q, \quad f(q)=q_{*},
$$


where exp* denotes the exponential map on the tangent bundle to $S^{n}\left(\pi^{2} / D^{2}\right)$. From the proof of Theorem 3.5.2 it is clear that $f$ is the desired diffeomorphism. The warped product structure of $M-\{p, q\}$ follows from Theorem 3.5.2 and Proposition 3.3.4. Q.E.D.

\section{BIBLIOGRAPHY}

1. Ralph Alexander, Planes for which lines are the shortest paths between points, Illinois J. Math. 22 (1978), 177-190.

2. Richard L. Bishop, Clairaut submersions, Differential Geometry, in Honor of K. Yano (S. Kobayashi, M. Obata and T. Takahashi, eds.), Kinokuniya Book-Store, Tokyo, Japan, pp. 21-31.

3. Richard L. Bishop and Barrett O’Neill, Manifolds of negative curvature, Trans. Amer. Math. Soc. 145 (1969), 1-49.

4. Luther Pfahler Eisenhart, Riemannian geometry, 2nd ed, Princeton Univ. Press, Princeton, N. J., 1949.

5. D. Hilbert and S. Cohn-Vossen, Geometry and the imagination (translated by Nemenyi), Chelsea, New York, 1952.

6. Tosio Kato, Perturbation theory for linear operators, 2nd ed., Grundlehren der Math. Wiss. 132 Springer-Verlag, New York, 1976.

7. Shoshichi Kobayashi and Katsumi Nomizu, Foundations of differential geometry, Vol. 1, Interscience Tracts in Pure and Appl. Math., no. 15, Interscience, New York, 1963.

8. Ravindra S. Kulkarni, Curvature and metric, Ann. of Math. (2) 91 (1970), 311-331.

9. R. G. Muhometov, The problem of recovery of a two-dimensional Riemannian metric and integral geometry, Soviet Math. Dokl. 18 (1977), 27-31.

10. Barrett O'Neill, The fundamental equations of a submersion, Michigan Math. J. 13 (1966), 459-469.

11. V. G. Romanov, Integral geometry on geodesics of an isotropic Riemannian metric, Soviet Math. Dokl. 19 (1978), 847-851.

12. Rainer Sachs and Hung-Hsi Wu, General relativity for mathematicians, Graduate Texts in Math., no. 48, Springer-Verlag, New York, 1977.

13. Michael Spivak, A comprehensive introduction to differential geometry, Vols. 1-5, Publish or Perish, Boston, Mass., 1975.

14. William Taber, Distance constraints and projectively equivalent metrics (in preparation).

15. Hermann Weyl, Zur infinitesimalgeometrie: Einordung der projektiven und der konfermen auffassung, Nachrichten von der Könglichen Gesellschaft der Wissenschaften zu Göttingen, Weidmannsche Buchhandling, Berlin, Germany, 1921.

DePartment of Mathematics, University of Illinois, Urbana, Illinois 61801

Current address: Jet Propulsion Laboratory, California Institute of Technology, Pasadena, California 91109 